\title{
A REMARK ON THE EQUATIONS OF AGE-DEPENDENT POPULATION DYNAMICS*
}

\author{
BY \\ MICHEL CHIPOT \\ Université de Nancy I
}

The aim of this note is to improve and complete the estimates given in [1]. We deal with the problem as introduced by M. E. Gurtin and R. C. MacCamy in [2], that is to say, we are looking for a function $\rho(a, t)$ satisfying

$$
\begin{cases}\rho_{a}(a, t)+\rho_{t}(a, t)+\mu(a, t, P(t)) \rho(a, t)=0 & \text { for } a, t>0, \\ \rho(0, t)=\int_{0}^{+\infty} \beta(a, t, P(t)) \rho(a, t) d a, & t>0, \\ \rho(a, 0)=\varphi(a), & a>0,\end{cases}
$$

where

$$
P(t)=\int_{0}^{+\infty} \rho(a, t) d a .
$$

As quoted in [1], the problem reduces to finding a function $\rho(a, t)$ which satisfies, for $a$, $t$ positive.

$$
\rho(a, t)=\left\{\begin{array}{l}
{\left[\int_{0}^{+\infty} \beta(\xi, t, P(t-a)) \rho(\xi, t-a) d \xi\right]} \\
\quad \cdot \exp -\int_{0}^{a} \mu(s, s+t-a, P(s+t-a)) d s \quad \text { if } a<t, \\
\varphi(a-t) \cdot \exp -\int_{a-t}^{a} \mu(s, s+t-a, P(s+t-a)) d s \quad \text { if } a>t,
\end{array}\right.
$$

the data being $\mu, \beta$ and $\varphi$. So, if $F \rho(a, t)$ denotes the right side of (2), the problem is to find a fixed point for $F$. In order to do this we will solve (2) for $(a, t) \in \mathbf{R}^{+} \times[0, T]$ and if $C(T)$ denotes a constant depending on $T$ only, we will assume:

$\mu(a, t, P)$ is a positive measurable function such that the mapping $s \rightarrow \mu(s, s+u, P)$ belongs to $L_{\mathrm{Loc}}^{1}([0,+\infty))$ for almost all $(u, P)$ in $\mathbf{R}^{2}$ and there exists a constant $C(T)$ such that, for all $P, P^{\prime} \in \mathbf{R}$,

${ }^{*}$ Received February 23, 1983. 


$$
\begin{aligned}
& \left|\mu(a, t, P)-\mu\left(a, t, P^{\prime}\right)\right| \leqslant C(T)\left|P-P^{\prime}\right| \text { a.e }(a, t) \in \mathbf{R}^{+} \times[0, T] . \\
& \beta(a, t, P) \text { is a measurable function and, for all } P, P^{\prime} \in \mathbf{R}, \\
& \left|\beta(a, t, P)-\beta\left(a, t, P^{\prime}\right)\right| \leqslant C(T)\left|P-P^{\prime}\right| \text { a.e. }(a, t) \in \mathbf{R}^{+} \times[0, T] .
\end{aligned}
$$

Now, the growth of $\beta$ has to be limited (see [1], [2]) and we assume: There exists a function $\bar{\beta}(P)$ continuous on $[0,+\infty)$ such that

$$
\begin{aligned}
& \bar{\beta}(P) \text { is nondecreasing, } \\
& \int_{A}^{+\infty} \frac{d s}{s \bar{\beta}(s)}=+\infty \quad \text { for some } A>0 \text { such that } \bar{\beta}(A)>0, \\
& |\beta(a, t, P)| \leqslant C(T) \bar{\beta}(|P|) \quad \text { a.e. }(a, t) \in \mathbf{R}^{+} \times[0, T] .
\end{aligned}
$$

Under these assumptions, which are better than in [1], we can prove

Theorem. If (3), (4), (5) hold for some $T$, then for each $\varphi \in L^{1}\left(\mathbf{R}^{+}\right)$there exists a unique $\rho \in L^{\infty}\left([0, T], L^{1}\left(\mathbf{R}^{+}\right)\right.$) satisfying (2) a.e. on $\mathbf{R}^{+} \times[0, T]$ ( $T$ can be taken arbitrarily if (3), (4), (5) hold for $T$ ).

The proof follows the same steps as in [1] and thus we only sketch it. First set

$$
G(t)=\int_{A}^{t} \frac{d s}{s \bar{\beta}(s)} .
$$

It follows from (5) that $G$ is a one-to-one mapping from $[A,+\infty)$ to $\mathbf{R}^{+}$. We will denote by $G^{-1}$ the inverse of $G$. Under the above assumptions, if ||$_{1}$ denotes the usual $L^{1}\left(\mathbf{R}^{+}\right)$norm and if $M=\operatorname{Max}\left(A,|\varphi|_{1}\right)$, we have

Lemma. If $\rho(a, t) \in L^{\infty}\left([0, T], L^{1}\left(\mathbf{R}^{+}\right)\right)$is a solution of (2), then

$$
|\rho(\cdot, t)|_{1} \leqslant G^{-1}[C(T) t+G(M)] \text { a.e. } t \in[0, T] .
$$

Proof. If $\rho$ satisfies (2), we get (using the fact that $\mu \geqslant 0$ )

$$
|\rho(\cdot, t)|_{1} \leqslant \int_{0}^{t}\left|\int_{0}^{+\infty} \beta(\xi, t, P(t-a)) \rho(\xi, t-a) d \xi\right| d a+\int_{t}^{+\infty}|\varphi(a-t)| d a .
$$

Hence after an easy computation using (5), we obtain

$$
|\rho(\cdot, t)|_{1} \leqslant|\varphi|_{1}+C(T) \int_{0}^{t} \bar{\beta}\left(|\rho(\cdot, s)|_{1}\right)|\rho(\cdot, s)|_{1} d s
$$

and thus

$$
|\rho(\cdot, t)|_{1} \leqslant M+C(T) \int_{0}^{t} \bar{\beta}\left(|\rho(\cdot, s)|_{1}\right)|\rho(\cdot, s)|_{1} d s=\psi(t) .
$$

As in [1, Lemma 1], there is no loss of generality in assuming $t \mapsto|\rho(\cdot, t)|_{1}$ continuous and thus from (7) and (5), we get

$$
\frac{d \psi(t)}{d t}=C(T) \cdot \bar{\beta}\left(|\rho(\cdot, t)|_{1}\right) \cdot|\rho(\cdot, t)|_{1} \leqslant C(T) \cdot \bar{\beta}(\psi(t)) \cdot \psi(t)
$$

i.e.

$$
\frac{d}{d t} G[\psi(t)] \leqslant C(T)
$$


Integrated between 0 and $t$ leads to

$$
G(\psi(t))-G(M) \leqslant C(T) t .
$$

Hence by (7) and the fact that $G^{-1}$ is nondecreasing

$$
|\rho(\cdot, t)|_{1} \leqslant \psi(t) \leqslant G^{-1}[C(T) t+G(M)] \text { a.e. } t \in[0, T] .
$$

This concludes the proof of the lemma.

The lemma entitles us to look for a solution $\rho$ of (2) in

$$
\begin{array}{r}
C=\left\{\rho \in L^{\infty}\left([0, T], L^{1}\left(\mathbf{R}^{+}\right)\right) \|\left.\rho(\cdot, t)\right|_{1} \leqslant G^{-1}[C(T) t+G(M)]\right. \\
\text { a.e. } t \in[0, T]\}
\end{array}
$$

and to conclude with the same proof as in [1]. That is to say, to prove that $F$ has a unique fixed point in $C$, we only need to prove that $F$ maps $C$ into $C$. So let $\rho \in C$. From (2) and with the same inequalities that we need in order to derive (7), we get for $t \in[0, T]$

$$
|F \rho(\cdot, t)|_{1} \leqslant M+C(T) \int_{0}^{t} \bar{\beta}\left(|\rho(\cdot, s)|_{1}\right)|\rho(\cdot, s)|_{1} d s .
$$

Thus by (5) and (8)

$$
\begin{aligned}
|F \rho(\cdot, t)|_{1} & \leqslant M+C(T) \int_{0}^{t} \bar{\beta}\left\{G^{-1}[C(T) s+G(M)]\right\} G^{-1}[C(T) s+G(M)] d s \\
& =M+\int_{0}^{t} \frac{C(T) d s}{G^{\prime}\left[G^{-1}(C(T) s+G(M))\right]} \\
& =M+\int_{0}^{t}\left(G^{-1}\right)[C(T) s+G(M)]^{\prime} d s=G^{-1}[C(T) t+G(M)] .
\end{aligned}
$$

This concludes the proof.

Remark. This result is the best possible in the sense that one cannot weaken the assumptions on the growth of $\beta$ and still get a global solution of (1). Indeed if we choose $\beta(a, t, P)=\bar{\beta}(P)$ with

$$
\int_{A}^{+\infty} \frac{d s}{s \bar{\beta}(s)}<+\infty
$$

then (see $[1,2])$ the resolution of (2) is equivalent, when $\mu \equiv 0, \varphi \geqslant 0$, to finding $P(t)$, a solution of

$$
\frac{d P(t)}{d t}=\bar{\beta}(P(t)) P(t), \quad P(0)=|\varphi|_{1},
$$

which in turn is equivalent to

$$
G(P(t))=t+G\left(|\varphi|_{1}\right) .
$$

But the solution of this problem blows up when $t+G\left(|\varphi|_{1}\right)$ goes out of the range of $G$, which is $\left[0, \int_{A}^{+\infty} d s / s \bar{\beta}(s)\right)$. 
Added in proofs: The above results can be improved limitating only the growth of $\beta-\mu$ (in $P$ ). See: M. C. in Proceedings of Journées d'Analyse Nonlineaire, Lille, France, May 1983.

\section{REFERENCES}

[1] M. Chipot, On the equations of age-dependent population dynamics Arch. Rat. Mech. Anal. 82, 1, 1983, pp. 13-25.

[2] M. E. Gurtin and R. C. MacCamy, Nonlinear age-dependent population dynamics Arch. Rat. Mech. Anal. 54 (1974), 281-300 